\title{
Understanding the antiangiogenic effect of metronomic chemotherapy through a simple mathematical model
}

\author{
Diego S. Rodrigues ${ }^{\mathrm{a}, *}$, Paulo F.A. Mancera ${ }^{\mathrm{a}}$, Suani T.R. Pinho ${ }^{\mathrm{b}, *}$ \\ ${ }^{a}$ Universidade Estadual Paulista "Júlio de Mesquita Filho", Instituto de Biociências, 18618-689, Botucatu, Brazil \\ ${ }^{\mathrm{b}}$ Universidade Federal da Bahia, Instituto de Física, 40210-340, Salvador, Brazil
}

\section{H I G H L I G H T S}

- A mean field model for antiangiogenic chemotherapy is proposed.

- Metronomic and maximum-tolerated schedules are compared by numerical simulations.

- Metronomic schedules are found to be more effective in eliminating tumour cells.

\section{A R T I C L E I N F O}

\section{Article history:}

Received 24 February 2016

Received in revised form 26 July 2016

Available online 9 August 2016

\section{Keywords:}

Cancer

Metronomic chemotherapy

Angiogenesis

Tumour growth

Differential equations

\begin{abstract}
A B S T R A C T
Despite the current and increasingly successful fight against cancer, there are some important questions concerning the efficiency of its treatment - in particular, the design of oncology chemotherapy protocols. Seeking efficiency, schedules based on more frequent, low-doses of drugs, known as metronomic chemotherapy, have been proposed as an alternative to the classical standard protocol of chemotherapy administration. The in silico approach may be very useful for providing a comparative analysis of these two kinds of protocols. In so doing, we found that metronomic schedules are more effective in eliminating tumour cells mainly due to their chemotherapeutic action on endothelial cells and that more frequent, low drug doses also entail outcomes in which the survival time of patient is increased.
\end{abstract}

(C) 2016 Elsevier B.V. All rights reserved.

\section{Introduction}

Cancer is considered a serious public health problem worldwide. One of the most commonly applied types of cancer treatment, antineoplastic chemotherapy, consists of the administration of one or more cycle-nonspecific or cycle-specific drugs whose aim is to destroy tumour cells. In general the aim of cancer therapies, including chemotherapy, is to maximize the therapeutic effect on tumours and minimize side effects on normal cells. Despite the advances in chemotherapy in recent decades, there lacks a better quantitative understanding of its mechanism. In order to improve and clarify how chemotherapy works, one can use mathematical modelling, for example, to design oncologic chemotherapy protocols (Panetta \& Fister [1]). Moreover, as we claim in this paper, mathematical modelling (as well as the in silico approach) may improve the comprehension of the antiangiogenic effect of metronomic chemotherapy.

Several methods have been employed to build mathematical models of tumour growth, such as ordinary and partial differential equations (Gatenby [2], Byrne \& Chaplain [3] and Chaplain et al. [4]), cellular automata (Moreira \& Deutsch

\footnotetext{
* Corresponding authors.

E-mail addresses: diegosarodrigues@gmail.com (D.S. Rodrigues), pmancera@ibb.unesp.br (P.F.A. Mancera), suani@ufba.br (S.T.R. Pinho).
} 
et al. [5]), optimization (Panetta \& Fister [1]) and multi-scale modelling (Stamatakos et al. [6], Macklin et al. [7] and Owen et al. [8]). Whatever approach is adopted, the combination of theory and data certainly provides the best results (Komarova [9]), where experiments guide theoretical models, and theoretical models lead to experiment(s) (Byrne et al. [10] and Araujo \& McElwain [11]). From this viewpoint, we develop, in this study, a simple mathematical model of tumour growth under chemotherapy, which was motivated by the biological experiment reported by Browder and collaborators [12]. Our aim is to describe and simulate the antiangiogenic character of an alternative chemotherapy protocol, with more frequent low drug doses per cycle, usually referred to as metronomic chemotherapy (Hanahan et al. [13] and Kerbel \& Kamem [14]).

Mathematical models (Hahnfeldt et al. [15] and Pinho et al. [16]) have been built to analyse a very relevant phenomenon for tumour growth extensively studied by Folkman's group [17]: tumour angiogenesis as well as antiangiogenic therapies for vascular tumours. The formation of new blood vessels (from a previous vascularization) due to the proliferation, migration and differentiation of endothelial cells that encase the blood vessels (Bikfalvi [18]) is essential for tumour growth after it reaches a diameter of $1-2 \mathrm{~mm}$ (vascular stage), and then the vascular stage is triggered by an angiogenic switch (Folkman [19]). This process induces a synthesis of several substances that stimulate the proliferation of new endothelial cells (Bussolino et al. [20]), which are not counterbalanced by small amounts of inhibitors (O'Reilly et al. [21]).

The inhibition of tumour angiogenesis may be promoted by antiangiogenic (non-chemotherapeutic) drugs or by the chemotherapeutic drug itself adopting, in comparison to standard protocols, more frequent, low-doses per cycle (metronomic chemotherapy). Using such a schedule of chemotherapy, Browder et al. [12] reported the elimination (drugsensitive tumours) or reduction (drug-resistant tumours) of Lewis lung carcinoma in mice, which did not occurr when a standard protocol was employed. Moreover, in some preclinical studies, metronomic chemotherapy is used to increase the survival time for human life (Bello et al. [22] and Man et al. [23]); for those, the cumulative drug doses are higher than those in standard schedules (keeping, however, the dose per infusion lower).

Baruchel \& Stempak [24] emphasize that metronomic protocols opened a new field of research and possibilities for patient treatment, but there are some questions related to the antiangiogenic efficiency of metronomic chemotherapy, such as its clear definition, the definition of optimum biological drug doses and the design of clinical trials. To corroborate Baruchel \& Stempak [24], Lien et al. [25] argue that low-dose metronomic chemotherapy can control tumour growth rate safely, but there are inconclusive phase III trial results so drug doses and intervals of drug infusion are empirical.

Motivated by both the reported experiments using metronomic chemotherapy and the open questions posed by Baruchel \& Stempak [24] and Lien et al. [25] related to its antiangiogenic efficiency, we intend to ascertain which features of that schedule are relevant to promoting a significant reduction in tumours. Our simple ordinary differential equation model simulates the administration of a chemotherapeutic cycle-nonspecific drug on a tumour, which is considered a homogeneous population of normal and tumour cells. Besides the competition between normal and tumour cells and the chemotherapeutic action on both of them, the model takes into account the chemotherapeutic action on vascular endothelial cells as well as the effect of angiogenesis on the carrying capacity of tumour cells. Our main purpose here is to compare one standard protocol of chemotherapy (which we will call the conventional schedule from now on) with metronomic chemotherapy, focusing on the antiangiogenic efficiency of metronomic chemotherapy in preventing tumoural neovascularization. We shall also address the effect on survival time entailed by metronomic chemotherapy.

The current paper is organized as follows: in Section 2, we present the model and some restrictions on its parameter values. In Section 3, we discuss the response of chemotherapy action according to the model; the numerical results of administration in cycles, simulating scenarios of standard and antiangiogenic protocols, showing in the latter an increase of both survival and log-kill reduction in tumour size. The limit case of continuous drug infusion is also discussed in Section 3 and analytically studied in the Appendix. Finally, Section 4 closes the paper with concluding remarks.

\section{The model}

We consider a compartment model formed by four nonlinear ordinary differential equations (ODE) whose compartments are normal cells, tumour cells, vascular endothelial cells and chemotherapeutic agent. Within the modalities of chemotherapy, we address one cycle-nonspecific drug under varying protocols: the standard protocol and the metronomic schedule (smaller cycle time intervals $T$ and lower dose-per-infusion than the conventional schedule) and the limit case of continuous drug infusion (i.e., not in bolus). Some parameter values of the model are based on experimental data for human tumours.

Although the angiogenic process inherently involves a spatial structure fully and explicitly analysed by partial differential equations (PDE) models (Chaplain et al. [4]), we consider the valuable approach of Hahnfeldt et al. [15] (see also d'Onofrio \& Gandolfi $[26,27])$. In it, a diffusion-like PDE for the factors that stimulate and inhibit tumoural angiogenesis is carefully reduced to an ODE in time that models the proliferation of vascular endothelial cells. Essentially, the modelling presented by Hahnfeldt et al. [15] and d'Onofrio \& Gandolfi [26] are used here to build a simple model which extends one of our previous models [28] by explicitly considering the compartment of endothelial cells as described in the sequel.

Tumour angiogenesis is represented in the model by the increase in carrying capacity of the tumour cells due to neovascularization. Namely, as we will present later, the increasing of the carrying capacity is directly related to the number of the vascular endothelial cells. To define the equation for vascular endothelial cells, as in Hahnfeldt et al. [15], we essentially consider the net production of both proangiogenic and angiogenic inhibition factors (Maggelakis [29]). The action of macrophages stimulating the release of angiogenic factors as well as the role of pericites in inhibiting the proliferation of 
endothelial cells (Levine et al. [30]) are contemplated through the angiogenic functions taken into account in the equation for vascular endothelial cells. Besides the net production of proangiogenic and angiogenic inhibition factors, we also consider, as in d'Onofrio \& Gandolfi [26], the average effect of the proliferation of endothelial cells within the tumour and their movement from the peritumoural region into the tumour as an additional effect. This is mainly a representation of a spatial effect: the diffusion of the endothelial cells itself. Despite the fact that metronomic chemotherapy has already been addressed via mathematical modelling in Hahnfeldt et al. [31] and Phipps [32], we did not find any simple ODE model that simultaneously includes metronomic chemotherapy and angiogenesis and that still considers the normal cell compartment and its interspecific competition with the tumour cells. As in Pinho et al. [33] and Nani \& Freedman [34], we include normal cells in the model since these are affected by the drug and also determine the maximum dose above which the drug becomes harmful to the oncologic patient.

Denoting the number of tumour cells and normal cells by $N_{i}(i=1,2)$, the number of vascular endothelial cells by $L_{1}$ and the dose of chemotherapeutic agent by $Q$, we propose the following model based on general functions as in Pinho et al. [35]:

$$
\left\{\begin{array}{l}
\frac{\mathrm{d} N_{1}}{\mathrm{~d} t}=r_{1} N_{1} g_{1}\left(N_{1}, L_{1}\right)-\mathcal{C}_{1}\left(N_{1}, N_{2}, L_{1}\right)-\mathscr{H}_{1}\left(N_{1}, Q\right), \\
\frac{\mathrm{d} N_{2}}{\mathrm{~d} t}=r_{2} N_{2} g_{2}\left(N_{2}\right)-\mathcal{C}_{2}\left(N_{1}, N_{2}, L_{1}\right)-\mathscr{H}_{2}\left(N_{2}, Q\right), \\
\frac{\mathrm{d} L_{1}}{\mathrm{~d} t}=\mathcal{A}\left(L_{1}\right)+s\left(N_{1}, L_{1}\right)-\ell\left(N_{1}, L_{1}\right)-\mathscr{H}_{3}\left(L_{1}, Q\right), \\
\frac{\mathrm{d} Q}{\mathrm{~d} t}=q(t)-\mathcal{W}\left(N_{1}, N_{2}, Q\right),
\end{array}\right.
$$

where $r_{i}, i=1,2$, are the growth rates associated with tumour cell and normal cell populations respectively, and the following functions:

- $g_{i}(\cdot) \geq 0$ represents the natural growth and its inhibition due to intraspecific competition;

- $\mathcal{C}_{i}(\cdot) \geq 0$ represents the interspecific competition between tumour and normal cells;

- $\mathcal{H}_{j}(\cdot) \geq 0(j=1,2,3)$ is the killing action of the drug on each cell population (in particular $j=3$ refers to vascular endothelial cell population);

- $s(\cdot) \geq 0$ models the ability to induce tumour vascularization, and therefore relates to the stimulator factors, and $\ell(\cdot) \geq$ 0 with respect to inhibitor factors;

- $\mathcal{A}(\cdot) \geq 0$ represents the proliferation of endothelial cells within the tumour and their migration from the peritumoural region into the tumour (d'Onofrio \& Gandolfi [26]);

- $q(t) \geq 0$, defined in $t \in[0, \infty)$, and $\mathcal{W}(\cdot) \geq 0$ model, respectively, the flux of the infusion of the chemotherapeutic drug, and the washout of the drug.

The non-autonomous dynamical system given by (1) is forced by the chemotherapeutic agent, whose administration is described below:

(i) Drug administration in cycles

In general, the drug is administered at fixed time intervals, and thus $q=q(t)$ is a periodic function. In this case, if the time spent for the infusion is much smaller than the total time of patient treatment, we assume that the drug interacts immediately with the tumour.

In standard human protocols, the period of each cycle is usually 3 weeks, followed by a rest period, in which chemotherapy is not applied. According to Martin \& Teo [36], we define the infusion rate as follows:

$$
q(t)=\left\{\begin{array}{c}
q_{\text {cycle }}>0, \quad n \leq t<n+\tau, \\
0, \quad n+\tau \leq t<n+T
\end{array}\right.
$$

where $T$ is the cycle time interval, $n=0, T, 2 T, \ldots$ and $\tau$ is the infusion time with $T \gg \tau$ (i.e., administration in "bolus" doses).

(ii) Continuous drug administration

In the limit case of continuous infusion, we assume small values for the infusion rate and $\tau \rightarrow T$. Therefore, the drug is infused at a constant rate: $q(t)=q>0, \forall t \geq 0$ with $q \ll q_{\text {cycle }}$

\subsection{Expliciting the model: selected functional responses}

Let us present the assumed functions for the general model (1).

(a) Cell growth functional response: $g_{i}(\cdot), i=1,2$ 
Although it seems that the growth of tumours does not follow a universal law (Retsky [37]), experimental results show it behaves as sigmoid curves like the generalized logistic function (Spratt et al. [38]) and the Gompertz function (Bajzer \& Pavlovic [39] and Michelson et al. [40]). We assume the logistic function for natural growth and intraspecific competition of tumour cells because it is more robust in fitting data for some human tumours according to Vaidya \& Alexandro Jr. [41]:

$$
g_{1}\left(N_{1}, L_{1}\right)=1-\frac{N_{1}}{k_{1}+L_{1}},
$$

where $k_{1}$ is the carrying capacity of the tumour in the pre-vascular stage. We call attention to the fact that the contribution to the carrying capacity of tumour cells due to tumour angiogenesis is represented by $L_{1}-$ the number of the vascular endothelial cells.

Analogously, we also assume the logistic function for natural growth and the intraspecific competition function for normal cells, but their carrying capacity is not affected by tumour angiogenesis, since we consider that normal cells are in homeostasis state (Hanahan \& Weinberg [42]):

$$
g_{2}\left(N_{2}\right)=1-\frac{N_{2}}{k_{2}},
$$

where $k_{2}$ is the carrying capacity of the normal cells.

(b) Interspecific cellular competition: $\mathcal{C}_{i}(\cdot), i=1,2$

The functions $\mathcal{C}_{i}(i=1,2)$ model the negative effect on tumour and normal cells due to interspecific competition for nutrients, as given by Gatenby [2]:

$$
\begin{aligned}
& \mathcal{C}_{1}\left(N_{1}, N_{2}, L_{1}\right)=r_{1} \frac{\alpha_{1}}{k_{1}+L_{1}} N_{1} N_{2}, \\
& \mathcal{C}_{2}\left(N_{1}, N_{2}, L_{1}\right)=\mathcal{C}_{2}\left(N_{1}, N_{2}\right)=r_{2} \frac{\alpha_{2}}{k_{2}} N_{1} N_{2},
\end{aligned}
$$

where $\alpha_{i}$ is the coefficient of the competition on the population $i$ and $r_{i}$ is the natural growth of population $i, i=1,2$ for tumour and normal cell populations.

(c) Angiogenic-related functional response: $\mathcal{A}(\cdot), \&(\cdot), \ell(\cdot)$

To model the temporal variation of vascular endothelial cells, we choose

$$
\begin{gathered}
\mathcal{A}\left(L_{1}\right)=\sigma L_{1}, \\
\&\left(N_{1}, L_{1}\right)=\phi N_{1}, \\
\ell\left(N_{1}, L_{1}\right)=\omega L_{1} N_{1} \xi,
\end{gathered}
$$

with $\mathcal{A}\left(L_{1}\right)$ assumed as a linear function of $L_{1}$ as proposed by d'Onofrio \& Gandolfi [26]; the parameter $\sigma$ is related to the proliferation of the endothelial cells within the tumour and their migration from the peritumoural region and therefore does not depend on the size of the tumour.

On the other hand, the net production of proangiogenic and angiogenic inhibition factors do depend on tumour size as stated by the functions $\delta\left(N_{1}, L_{1}\right)$ and $\ell\left(N_{1}, L_{1}\right)$. The function $\ell(\cdot)$ that is presented here does not allow an exponential tumour growth, exactly as discussed by d'Onofrio \& Gandolfi [26]. The larger the tumour, the more poorly perfused and chaotic the tumoural neovasculature will be (Boareto et al. [43]), because of the difficulty to reach the centre of the tumour (where the normoxic cells are) scales as its size and because of the microvessels inefficiently trend of decreasing its diameter as they have to increasingly reach a more vast portion of the tumour volume. The function $\delta(\cdot)$ is a mathematical consequence of the biological assumption that the angiogenesis stimulus only depends on the tumour size. Both $\S\left(N_{1}, L_{1}\right)$ and $\ell\left(N_{1}, L_{1}\right)$ functions are based on Hahnfeldt et al. [15] modelling, where for the sake of analyticity we set $\xi=1$. Moreover, $\phi$ measures the angiogenic factors released by the tumour, whereas $\omega$ models the neo-vascularization inhibition by the tumour itself.

(d) Drug functional response: $\mathcal{W}(\cdot), \mathscr{H}_{i}(\cdot), i=1,2,3$

For intravenous application of the drug, temporal variation in the amount of the drug is given by first order kinetics, and so $\mathcal{W}\left(N_{1}, N_{2}, Q\right)$ is just a function of $Q$, as usually considered (Hahnfeldt et al. [15] and Lüllmann et al. [44]) in pharmacokinetics. Therefore, it follows that

$$
W(Q)=\lambda Q
$$

where $\lambda>0$ is the washout rate of a given cycle-nonspecific chemotherapeutic drug.

Regarding the drug effect, i.e. pharmacodynamics, we assume the classical log-kill functional response (i.e. the percentage of cells eliminated by the drug is always the same constant) hypothesized by Skipper et al. [45], with a saturation of the Michaelis-Menten type for the amount of the drug. Therefore $\mathscr{H}_{1}(\cdot), \mathscr{H}_{2}(\cdot)$ and $\mathscr{H}_{3}(\cdot)$ are given by

$$
\mathscr{H}_{1}\left(N_{1}, Q\right)=\frac{\mu N_{1} Q}{a+Q},
$$




$$
\begin{aligned}
& \mathscr{H}_{2}\left(N_{2}, Q\right)=\frac{v N_{2} Q}{b+Q}, \\
& \mathscr{H}_{3}\left(L_{1}, Q\right)=\frac{\eta L_{1} Q}{c+Q},
\end{aligned}
$$

where $a, b$ and $c$ determine the saturation of the drug functional response (they are the amount of the drug for which the respective effect is half of its maximum), $\mu$ is the treatment rate of the tumour cells, $v$ is the mortality rate of normal cells due to treatment and $\eta$ models the intensity of the antiangiogenic effect of the chemotherapeutic drug.

Pinho et al. [35] proposed a general saturated functional response for $\mathscr{H}_{j}\left(N_{i}, Q\right)$ in which saturation occurs both in $Q$ and $N_{i}$. Particular cases are considered in Ref. [33] (saturation only in $N_{i}$ ) and in Ref. [35] (saturation only in $Q$ ). In the present study, we follow the second simplification of the functional response, which properly describes the saturation effect of the drug as in Aroesty et al. [46].

Finally, replacing (3)-(13) in the general model (1), we obtain the model that we will assume:

$$
\left\{\begin{array}{l}
\frac{\mathrm{d} N_{1}}{\mathrm{~d} t}=r_{1} N_{1}\left(1-\frac{N_{1}}{k_{1}+L_{1}}-\frac{\alpha_{1} N_{2}}{k_{1}+L_{1}}\right)-\frac{\mu N_{1} Q}{a+Q}, \\
\frac{\mathrm{d} N_{2}}{\mathrm{~d} t}=r_{2} N_{2}\left(1-\frac{N_{2}}{k_{2}}-\frac{\alpha_{2} N_{1}}{k_{2}}\right)-\frac{\nu N_{2} Q}{b+Q}, \\
\frac{\mathrm{d} L_{1}}{\mathrm{~d} t}=\sigma L_{1}+\phi N_{1}-\omega N_{1} L_{1}-\frac{\eta L_{1} Q}{c+Q}, \\
\frac{\mathrm{d} Q}{\mathrm{~d} t}=q(t)-\lambda Q .
\end{array}\right.
$$

with $q(t)$ given by (2). All parameter values in (14) are non-negative except for $\lambda, k_{1}$ and $k_{2}$, which are strictly positive. This model extends a previous one studied by us in Ref. [28]: a limit case for which angiogenesis reaches equilibrium before the drug takes action, and when there is no antiangiogenic effect of the drug at all $(\eta=0)$. We can interpret the former assertion assuming that $L_{1}$ is a population of vascular endothelial cells is in a steady state; let us denote such a constant value of cells by $L_{1}^{\infty}$. In such a case, we reinterpret the carrying capacity $k_{1}$ as $\widetilde{k}_{1}=k_{1}+L_{1}^{\infty}$. Numerical simulations verify that this is the case and they are shown in Ref. [47].

\subsection{Restrictions on parameter values}

Based on pharmacological and biological arguments, we make some restrictions on the following parameter values.

Regarding growth rates, the tumour grows faster than normal tissues because the tumour cells are able to delay apoptosis (programmed cell death). If $r_{i}$ is given by $r_{i}=b_{i}-d_{i}$, where $b_{i}$ is the rate of cell division and $d_{i}$ is the rate of cell death, then from a biological viewpoint $b_{1} \approx b_{2}$ but, due to tumour cells' delay of apoptosis, $d_{1}<d_{2}$, implying

$$
r_{1}>r_{2}
$$

and although there is such inequality, it is noteworthy that tumours in different bodily regions have different values for $r_{1}$, and each type of normal cell has a value of $r_{2}$.

In relation to the angiogenic process, from Eq. (14) we have

$$
\phi \gg \sigma
$$

because we expect that the proliferation due to stimulator factors is more significant than the effect of adjacent endothelial cells and their migration from the peritumoural region into the tumour. Moreover, the inhibition rate becomes comparable to the proangiogenic rate only when $L_{1}$ is relatively large such that $\omega L_{1} \approx \phi$. For such a reason, we assume that the following inequality holds:

$$
\omega \max \left\{L_{1}\right\} \lesssim \phi .
$$

Finally, for the treatment to make sense, the chemotherapeutic drug must show greater efficiency in killing tumour cells than normal cells. For example, according to Buick [48], the drug effect in lymphomas is up to $10^{4}$ times higher than in bone marrow cells, where we have

$$
\mu \gg v \text {. }
$$

Besides, it is important to guarantee that, in the special case of non-treated scenario the tumour is not eliminated, as in Pinho et al. [33] and Nani \& Freedman [34]. However, here it is allowed that system (14), with $Q(t) \equiv 0$ for any time $t$, may reach the coexistence equilibrium between normal and tumour cells. The four equilibria $E_{i}\left(\hat{N}_{1}, \hat{N}_{2}, \hat{L}_{1}\right), i=1,2,3,4$, of system (14) with $Q(t) \equiv 0$ are given by

- $E_{1}(0,0,0)$-Extinction of normal, endothelial and tumour cells;

- $E_{2}\left(0_{\sim} k_{2}, 0\right)$-Extinction of tumour cells (spontaneous cure);

- $E_{3}\left(N_{1}, 0, L_{1}\right)$-Extinction of normal cells in the presence of tumour cells and endothelial vascular cells;

- $E_{4}\left(N_{1}^{*}, N_{2}^{*}, L_{1}^{*}\right)$-Coexistence of cells. 


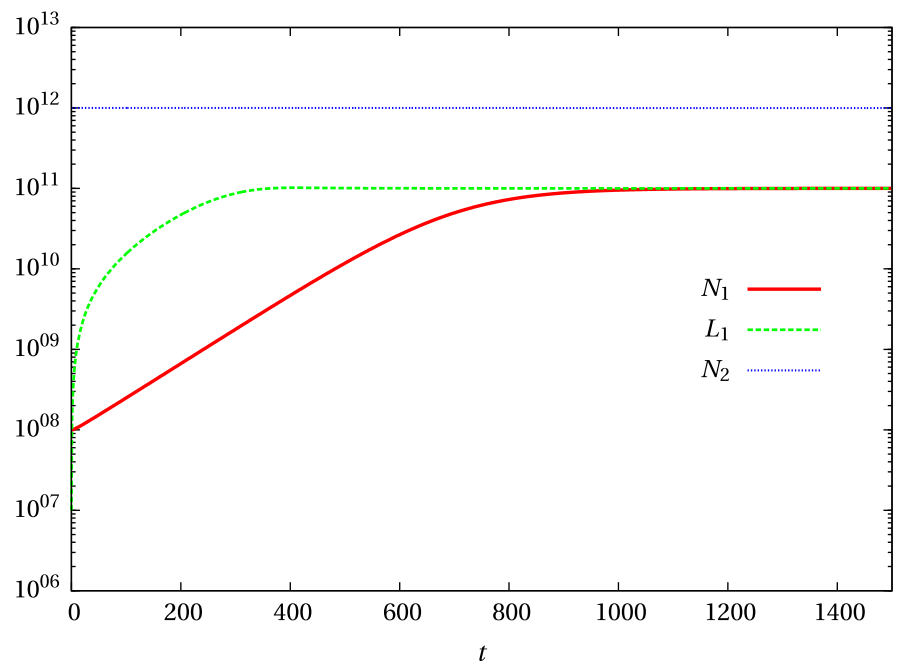

Fig. 1. Tumour evolution in absence of therapies (numerical integration of system (14) with $Q(t) \equiv 0)$ ). Initial condition: $N_{1}(0)=10^{8}$ tumour cells (red solid line), $N_{2}(0)=10^{12}$ normal cells (blue dashed line) and $L_{1}(0)=10^{7}$ vascular endothelial cells (green dashed line). Parameters values are from Table 1 . (For interpretation of the references to colour in this figure legend, the reader is referred to the web version of this article.)

It is easy to show that the non-biological equilibrium $E_{1}(0,0,0)$ and disease free equilibrium $E_{2}\left(0, k_{2}, 0\right)$ are locally unstable for $\sigma>0$ and any non-negative values of the other parameters (except for $k_{1}>0$ and $k_{2}>0$ ). If we assume $\sigma=0$, we have to impose that

$$
k_{1}>\alpha_{1} k_{2}
$$

in order to guarantee the unstable character of $E_{2}\left(0, k_{2}, 0\right)$ (this condition is obeyed in the numerical simulations). It is easy to prove that, without treatment, the system may evolve to $E_{4}\left(N_{1}^{*}, N_{2}^{*}, L_{1}^{*}\right)$ in the positive cone, if

$$
\alpha_{1} \alpha_{2}<1 \text { and } \frac{\sigma}{\omega}<N_{1}^{*}<\frac{k_{2}}{\alpha_{2}} \text {. }
$$

Also,

$$
\begin{aligned}
N_{1}^{*} & =\frac{k_{1}+L_{1}^{*}-\alpha_{1} k_{2}}{1-\alpha_{1} \alpha_{2}}, \\
N_{2}^{*} & =k_{2}-\alpha_{2} N_{1}^{*}, \\
L_{1}^{*} & =\frac{\phi}{\omega-\frac{\sigma}{N_{1}^{*}}} .
\end{aligned}
$$

Eqs. (21) and (22) indicate that, being on the equilibrium state, the increasing of the tumour $\left(N_{1}\right)$ only occurs by the augmentation of vascularization $\left(L_{1}\right)$. This is completely expected, but our model materializes such a biological phenomenon into a mathematical idea.

To conclude this section we present a numerical simulation when no therapy is applied, where the numerical integration of the system (14) is obtained by using Runge-Kutta 4th order method. We access the "angiogenic switch" phenomenon reported by Folkman [19], where the augmentation of vascular endothelial growth factor essentially triggers the tumoural angiogenesis. The curves for $N_{1}$ and $L_{1}$ versus time in Fig. 1 reveal such a phenomenon by means of a time lag between tumour cells and endothelial cells growth. By a disbalance between the neovasculature stimulus and inhibition, the former is more pronounced than the latter, thus the tumoural angiogenesis is triggered. The number of normal cells remains at the same amount as in the beginning of the dynamics, because of its homeostasis initial state. All the previous inequalities related to parameters restrictions hold in the results of Fig. 1. The parameter values for such a non-treated case are listed in Table 1.

\section{Results and discussion}

In this section, we analyse the model (14) taking into account the administration in cycles of a chemotherapeutic drug by using an in silico approach to reach the main point of our paper: understanding the antiangiogenic effect of metronomic chemotherapy. After calibrating the model in a scenario of absence of therapies (Fig. 1) we simulate a standard protocol of chemotherapy and many types of metronomic protocols. Finally, we discuss the limit case of continuous drug administration based on some analytical results and simulations. 
Table 1

Parameters related to the tumour, normal and endothelial cells for model (14).

\begin{tabular}{llll}
\hline Parameter & Value & Unity & Reference/Comments \\
\hline$r_{1}$ & $10^{-2}$ & day $^{-1}$ & Spratt et al. [38] \\
$r_{2}$ & $10^{-3}$ & day $^{-1}$ & See (15) \\
$k_{1}$ & $10^{8}$ & cell & Spratt et al. [38], Kerbel [49] \\
$k_{2}$ & $10^{12}$ & cell & Weinberg [50], Schaebel [51] \\
$\alpha_{1}$ & $9 \times 10^{-5}$ & - & See (19) \\
$\alpha_{2}$ & $9 \times 10^{-2}$ & - & - \\
$\sigma$ & $10^{-3}$ & day $^{-1}$ & $\sigma \sim r_{2}$ \\
$\phi$ & 1 & day $^{-1}$ & See $(16)$ \\
$\omega$ & $10^{-11}$ & cell $^{-1}$ day $^{-1}$ & See $(17)$ \\
\hline
\end{tabular}

${ }^{a}$ Per Spratt et al. [38] a 6 mm-diameter tumour has $1.13 \times 10^{8}$ cells, and Kerbel [49] affirms that an avascular tumour has a diameter less than or equal to $2 \mathrm{~mm}$.

b The carrying capacity of normal cells $k_{2}$ can also be estimated considering that $10^{9}$ cells equals $1 \mathrm{~g}$ [52] and that a human adult has about $10^{13}$ cells $[53,51]$.

Table 2

Parameters related to the chemotherapeutic action for model (14).

\begin{tabular}{|c|c|c|c|}
\hline Parameter & Value & Unity & Reference/Comment \\
\hline$v$ & $8 \times 10^{-2}$ & day $^{-1}$ & $v>r_{2}$ \\
\hline$\mu$ & 8 & day $^{-1}$ & See (18) \\
\hline$\lambda$ & 4.16 & day $^{-1}$ & {$[54]^{\mathrm{a}}$} \\
\hline$a$ & $2 \times 10^{3}$ & $\mathrm{mg}$ & Assumed value ${ }^{\mathrm{b}}$ \\
\hline$b$ & $5 \times 10^{6}$ & $\mathrm{mg}$ & Assumed value $\mathrm{b}^{\mathrm{b}}$ \\
\hline$c$ & $2 \times 10^{3}$ & $\mathrm{mg}$ & $c \sim a$ \\
\hline
\end{tabular}

\subsection{Comparative analysis between conventional and metronomic chemotherapy}

Assuming the administration in cycles given by (2), in order to access the antiangiogenic effect of chemotherapy, we simulate different schedules, varying the intensity of the dose per infusion $q_{\text {cycle }}$ and the cycle time interval $T$. The known discontinuities in $q(t)$ were circumvented by the mere inclusion of an if-statement in the Runge-Kutta code. Also, $Q(0)$ was set equal to 0 in all simulations. In Table 2 we summarize the parameters related to chemotherapeutic action.

An oncologic protocol established for breast cancer treatment is FEC100 [55] (fluorouracil, epirubicin and cyclophosphamide) and requires, among other drugs, the intravenous application of cyclophosphamide in bolus at a dose of $500 \mathrm{mg} / \mathrm{m}^{2}$ of the body surface of the patient every 21 days. We adopt this oncologic protocol, but considering just cyclophosphamide in the treatment. This is because synergistic and antagonistic drug-drug interactions are not easy to model (they can also depend on the drugs used).

From a formula given by Mosteller [56], we estimate that the body surface of a patient with a weight of $70 \mathrm{~kg}$ and height $1.70 \mathrm{~m}$ is $1.8 \mathrm{~m}^{2}$, thus establishing a dose of $900 \mathrm{mg}$ per cycle. In fact, the administration time of cyclophosphamide is much less than $3 \mathrm{~h}$. However, we assume that this dose is infused over $3 \mathrm{~h}$ because we assume that the drug interacts immediately with the tumour and also because cyclophosphamide's peak plasma concentration occurs approximately $3 \mathrm{~h}$ after infusion [54]. An infusion of $3 \mathrm{~h}$ (1/8 day) implies an infusion rate of $8 \times 900=7200 \mathrm{mg} / \mathrm{day}$. This corresponds to a standard protocol which, for our purpose, will be called the conventional schedule. From (2), we define such a regimen by

$$
q(t)= \begin{cases}7200, & l \leq t<l+\frac{1}{8} \\ 0, & l+\frac{1}{8} \leq t<l+21\end{cases}
$$

where $l=0,21,42,63$ ( $n_{\text {inf }}=4$ infusions), with $q(t \geq 84) \equiv 0$.

We stress that, for metronomic chemotherapy, the values of $q_{\text {cycle }}$ and $T$ are reduced in relation to the conventional schedule (which means that the doses per cycle are lower and the drug is administered more frequently). To highlight the differences, we present Table 3.

In order to compare the effect of the conventional schedule and metronomic chemotherapy on tumoural reduction, and remembering that the total dose is given by $D=q_{\text {cycle }} n_{\text {inf }} \tau$, we set up the two case studies described below.

Note: the number of normal cells was omitted in the results since the treatment had almost no effect on it in all cases (lower cytotoxic level of drug) due to the lower value of $v$ in comparison to $\mu$; in other words, the order of magnitude remained at $10^{12}$ normal cells during the whole treatment in all cases. 
Table 3

Parameters related to chemotherapy administration in cycles for model (14); chemotherapeutic drug: cyclophosphamide.

\begin{tabular}{lll}
\hline Parameter & Conventional & Metronomic \\
\hline Drug infusion rate $\left(q_{\text {cycle }}\right)$ & $7200 \mathrm{mg} /$ day & $q<7200 \mathrm{mg} /$ day \\
Cycle time interval $(T)$ & 21 days & $T<21$ days \\
Number of infusions $\left(n_{\text {inf }}\right)$ & 4 & $n_{\text {inf }}>4$ \\
Drug infusion time $(\tau)$ & $1 / 8$ day $=3 \mathrm{~h}$ & $1 / 8$ day $=3 \mathrm{~h}$ \\
Total drug dose $\left(D=q_{\text {cycle }} n_{\text {inf }} \tau\right)$ & $D_{\mathrm{c}}=3600 \mathrm{mg}$ & $D \geq 3600 \mathrm{mg}$ \\
Antiangiogenic effect on endothelial cells $(\eta)$ & $\eta>0$ day $^{-1}$ & $\eta>0$ day $^{-1}$ \\
\hline
\end{tabular}

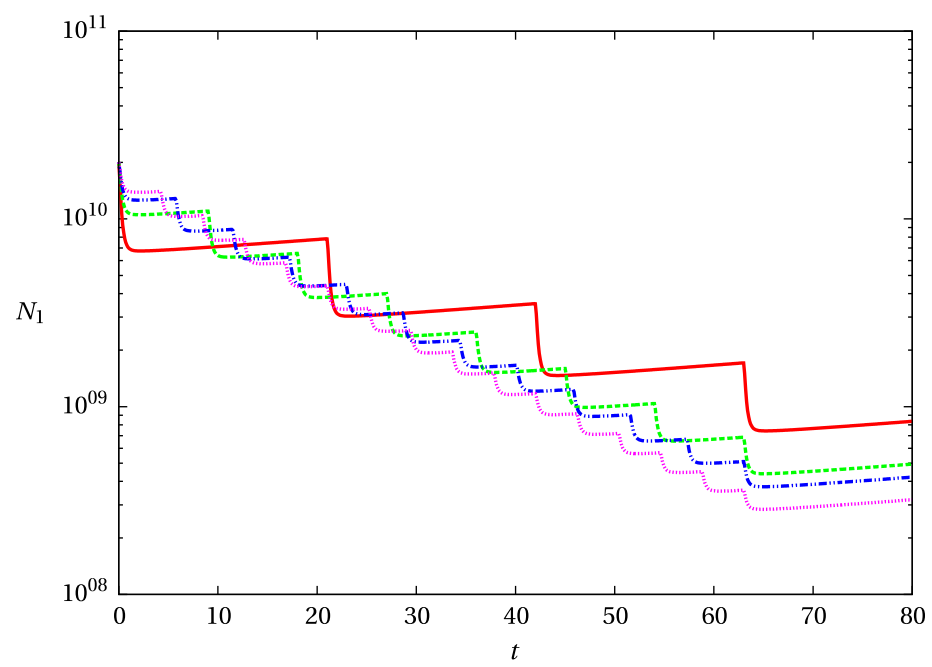

Fig. 2. Number of tumour cells for periodic chemotherapy treatment for non-null chemotherapeutic action on vascular endothelial cells $(\eta \neq 0$ ). Conventional (red thickest solid line) and metronomic schedules (the three thinnest dashed lines, correspond to $n_{\text {inf }}=16,12$ and 8 ) until 80 days (the last day of infusion is the 63rd). Initial condition: $N_{1}(0)=2 \times 10^{10}, N_{2}(0)=10^{12}, L_{1}(0)=10^{2}, Q(0)=0$; the parameter values are those presented in Tables 1-3, with $\eta=500$ day $^{-1}$. (For interpretation of the references to colour in this figure legend, the reader is referred to the web version of this article.)

\subsubsection{CASE STUDY I-computing the antiangiogenic effect}

CASE STUDY I summary: we set both total dose $D$ at $D_{\mathrm{c}}=3600 \mathrm{mg}$ and the total time of treatment (63 or 42 days, depending on the case) but make $q_{\text {cycle }}, T$ and $n_{\text {inf }}$ schedule-specific. For either 42 or 63 days we simulate three metronomic schedules with $n_{\text {inf }}$ equal to 8, 12 or 16 infusions. Let us clarify that Figs. 2 and 3 are related to the schedule of 63 days whereas Table 4 is relative to the 42 -day-schedules.

As we can see in Fig. 2, the lower and the more frequent the dose, the greater the tumoural reduction will be. For the same tumoural curves, the lowest value of tumour cells reached during each respective protocol is:

- Conventional schedule: $7.5 \times 10^{8}$ tumour cells;

- Metronomic chemotherapy:

- $n_{\text {inf }}=8$ and $T=9$ days: $4.4 \times 10^{8}$ tumour cells;

- $n_{\text {inf }}=12$ and $T=63 / 11 \approx 5.72$ days: $3.7 \times 10^{8}$ tumour cells;

- $n_{\text {inf }}=16$ and $T=63 / 15=4.2$ days: $2.8 \times 10^{8}$ tumour cells.

Therefore, fixing the total dose (i.e. with the same total amount of drug), we have shown that the shorter the interval between doses is, the greater the tumoural reduction will be. However, how exactly this effect is related to endothelial cells? In order to answer this question, we deactivate the effect of the chemotherapeutic drug on endothelial cells by setting $\eta=0$. In so doing, we can separate the effect of cycle time interval reduction on the tumour from the antiangiogenic effect on endothelial cells. Intuitively, one would expect that the smaller cycle time interval $T$ the smaller the tumour will become (because the tumour would have less time to regrow between infusions). Surprisingly, however, without the antiangiogenic effect $(\eta=0)$, a smaller cycle time interval $T$ does not imply a smaller tumour: as we show in Table 4, if $T=6$ days, the lowest value is $1.1 \times 10^{9}$ tumour cells, which is the same if $T=42 / 11 \approx 3.81$ days. This is mainly an indication of the essential role that the antiangiogenic chemotherapy effect can play; such an effect is essential to lead to a greater tumoural reduction: when $\eta=500$ day $^{-1}$, the number of tumour cells is still lower, as we make evident in Table 4. Inspired by experimental tumour data of drug-sensitive Lewis lung carcinoma in mice (Browder et al. [12]), we have simulated the model assuming a value of $\eta\left(\eta=500 \mathrm{day}^{-1}\right)$ for which the conventional schedule does not have an antiangiogenic effect strong enough to reduce the tumour to values smaller than a clinically detectable tumour size $\left(10^{9}\right.$ cells $)$. Using such a 
Table 4

Analysis of the cycle time interval $T$ excluding from the antiangiogenic chemotherapeutic effect itself. Total treatment time: 42 days; total dose: $D=3600 \mathrm{mg}$.

\begin{tabular}{clcl}
\hline$n_{\text {inf }}$ & $T$ (days) & $\eta\left(\right.$ day $\left.^{-1}\right)$ & Minimum of tumour cells \\
\hline \multirow{2}{*}{8} & 6 & 0 & $1.1 \times 10^{9}$ \\
& & 500 & $3.8 \times 10^{8}$ \\
12 & 3.81 & 0 & $1.1 \times 10^{9}$ \\
& & 500 & $3.2 \times 10^{8}$ \\
16 & 2.8 & 0 & $0.9 \times 10^{9}$ \\
& & 500 & $2.5 \times 10^{8}$ \\
\hline
\end{tabular}

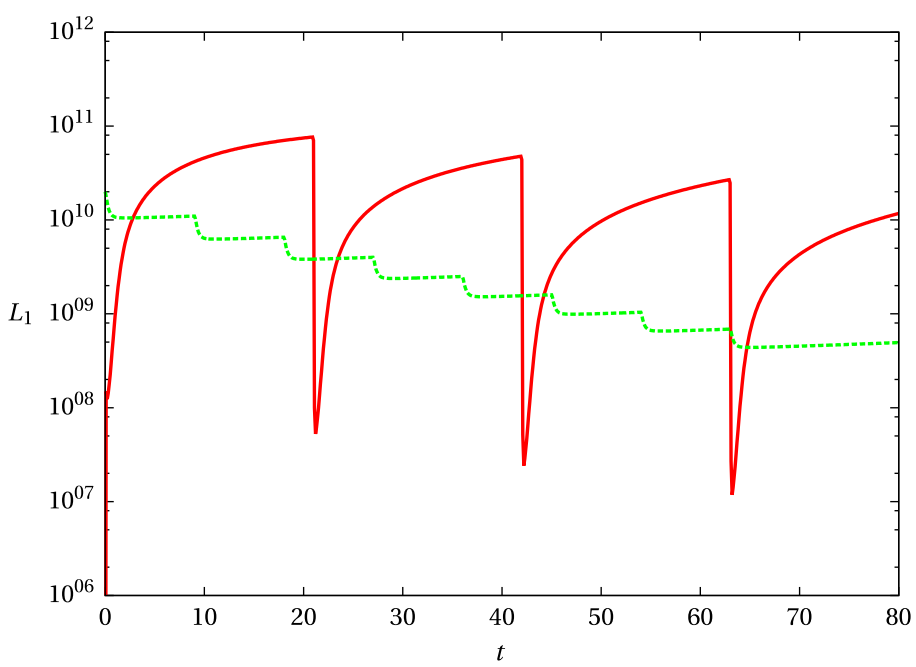

Fig. 3. Number of vascular endothelial cells for a conventional schedule (red thickest solid line) and for metronomic chemotherapy schedule with $n_{\text {inf }}=8$ (green thinnest dashed line). The $L_{1}$ curve corresponds to the same simulation presented in Fig. 2 (in which we plot $N_{1}$ curves). The schedules for $n_{\text {inf }}=12$ and $n_{\text {inf }}=16$ were omitted for a better visualization. (For interpretation of the references to colour in this figure legend, the reader is referred to the web version of this article.)

value for the antiangiogenic effect, however, enables our metronomic schedules to get smaller non-clinically detectable tumours.

Comparing the evolution of vascular endothelial cells for the conventional schedule and a metronomic one in which we set $n_{\text {inf }}=8$, it is very clear that increasing the frequency of low doses enhances the antiangiogenic effect of the chemotherapeutic drug (Fig. 3).

According to this case study, fixing the dose per cycle and the cytotoxic action of chemotherapeutic drug on tumour cells, it is important that the antineoplastic drug - which is not antiangiogenic but can promote this additional effect when one uses more frequent, low doses - acts efficiently on endothelial cells. That importance lies in the additional tumoural reduction arising from such an action. Moreover, we observe that if there is no antiangiogenic effect, decreasing the time interval $T$ between drug infusions may not lead to smaller tumours, as is clearly shown in Table 4.

Before we move on to CASE STUDY II, we make here a brief comment about the deviation from metronomic scheduling, i.e., is there any advantage in varying the time interval $T$ during treatment? Consider a sequence of cycles for which the time interval between doses is gradually reduced, maintaining the total dose as well as the total treatment time. Numerical simulations (not shown) do not reveal any relevant advantage in tumour reduction at the end of treatment in relation to a fixed period $T$.

\subsubsection{CASE STUDY II-Antiangiogenic protocols and human survival time}

CASE STUDY II summary: we compare a conventional schedule and a metronomic one, both with total doses $D_{\mathrm{c}}$ and $2 D_{\mathrm{c}}$ performing a total of four protocols. Our goal here is to investigate the differences between them with respect to the argument that metronomic chemotherapy increases human survival time. In particular, two out of our four protocols are based on Browder et al. [12], where the authors define the total dose of an antiangiogenic schedule as being twice that used in a standard one (or in our terminology, the conventional schedule at dose $D_{\mathrm{c}}$ and the metronomic one in which the dose is $2 D_{\mathrm{c}}$ ).

In Fig. 4 we display the time evolution curves of tumour cells for the four situations in CASE STUDY II, whose main information is summarized in Table 5. Note that, unlike the conventional schedule at dose $D_{c}$, for the other three cases, the number of tumour cells is reduced from an order of magnitude of $10^{10}$ cells to less than $10^{9}$ cells, that is, they become 


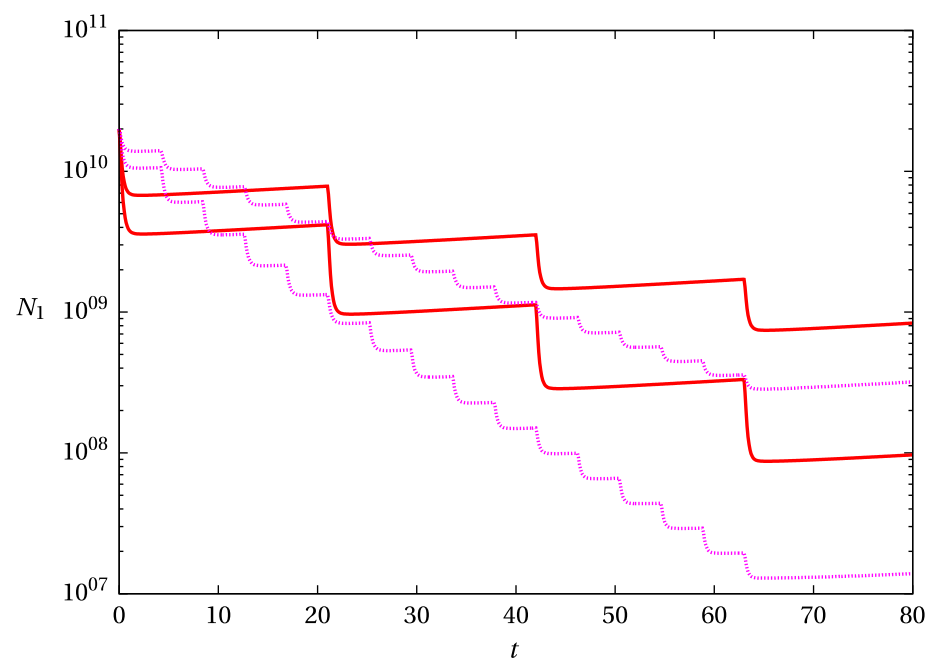

Fig. 4. Number of tumour cells for conventional schedule with dose $D_{\mathrm{c}}=3600 \mathrm{mg}$ and $2 D_{\mathrm{c}}=7200 \mathrm{mg}$ (red thickest solid lines), metronomic chemotherapy with dose $D_{\mathrm{c}}=3600 \mathrm{mg}$ and $2 D_{\mathrm{c}}=7200 \mathrm{mg}$ (magenta thinnest dashed lines). (For interpretation of the references to colour in this figure legend, the reader is referred to the web version of this article.)

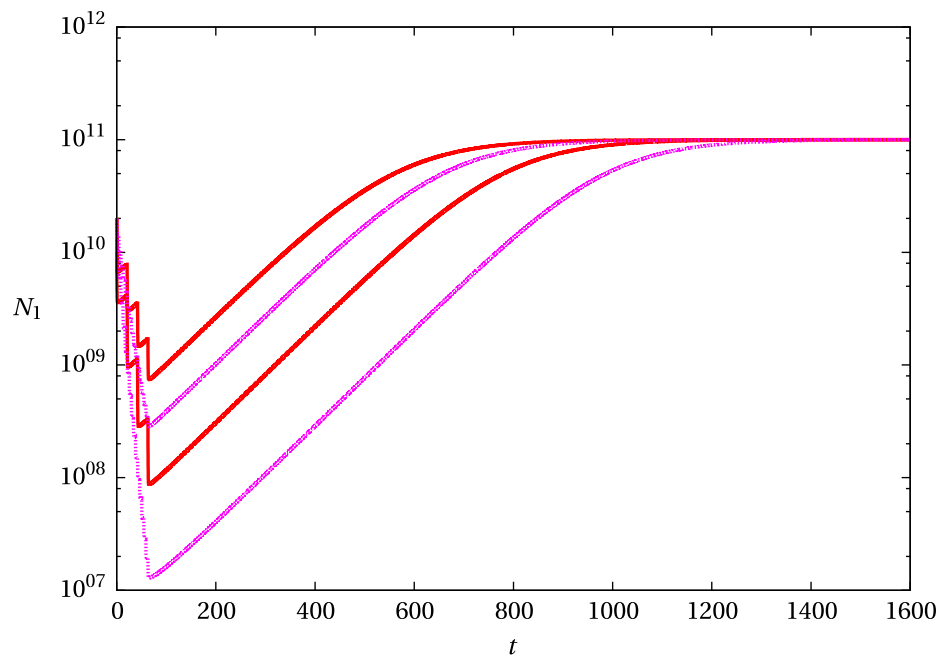

Fig. 5. The same curves of Fig. 4, but for $t=1800$ days. Parameter values are presented in Tables $1-3$, with $\eta=500$ day $^{-1}$.

clinically undetectable tumours. By the same simulation, but for a longer time of 1600 days, in Fig. 5, it is possible to analyse the survival time assuming that the patient does not undergo any other treatment after the schedule itself. By survival time we mean the time interval in which the tumour reaches $10^{11}$ cells that corresponds to tumoural mass of $100 \mathrm{~g} .{ }^{1}$ In Table 5 , we point out in numbers such a comparison among the schedules. We can clearly see the impact of the metronomic effect on the patient's survival time: it results in $\approx 100$ more days of life if the dose is $D_{\mathrm{c}}$ and in an additional survival time of $\approx 220$ more days if the drug dose is $2 D_{c}$.

Therefore, according to Figs. 4 and 5, both facts are relevant for increasing the patient's survival time as was pointed out by Bello et al. [22] and Man et al. [23]. Of course, it is not surprising that increasing the total drug dose, assuming the chemotherapeutic drug effect is almost non-cytotoxic on normal cells, the time survival of the patient also increases, even in the conventional schedule. The main point here is that the antiangiogenic metronomic schedules lead to a smaller minimum tumour size (below $10^{8}$ cells in Fig. 4 and Table 5). That is a good outcome because other treatments may be applied in order to avoid tumour regrowth as it is shown in Fig. 5.

\footnotetext{
1 In fact a breast tumour greater than $5 \mathrm{~cm}\left(\approx 100 \mathrm{~g}\right.$ or with $10^{11}$ cells $)$ can be life-harmful according to the American Joint Committee on Cancer Staging System for Breast Cancer [57].
} 
Table 5

Analysis of patient's survival time after early ceasing chemotherapy.

\begin{tabular}{lllr}
\hline Total dose $(\mathrm{mg})$ & Schedule & Minimum of tumour cells & Survival time (days) \\
\hline $3600\left(D_{\mathrm{c}}\right)$ & Conventional & $7.4 \times 10^{8}$ & 780 \\
$3600\left(D_{\mathrm{c}}\right)$ & Metronomic & $2.8 \times 10^{8}$ & 880 \\
$7200\left(2 D_{\mathrm{c}}\right)$ & Conventional & $8.7 \times 10^{7}$ & 1000 \\
$7200\left(2 D_{\mathrm{c}}\right)$ & Metronomic & $1.3 \times 10^{7}$ & 1220 \\
\hline
\end{tabular}

\subsection{The limit case of continuous drug administration}

Although our focus is on the periodic schedule, motivated by the cases where the drug is administered continuously, we analyse the limit case for which $\tau \rightarrow T$ and $q(t)=q$ for any $t \geq 0$, taking advantage of the process to obtain some analytical results as well. Of course, in real cases, the dose is applied until a certain time $t_{\text {end }}$, i.e. for a finite value of $n$, leading to a Heaviside function. For our analysis, we assume a very small dose per cycle infusing for a very long time and reaching the same total dose for the Heaviside infusion.

In this case, for some ranges of parameter values, system (14) may evolve to a cure state where there are no tumour cells. Since we have observed in the previous section that the most relevant parameter is $\eta$, the killing action of a chemotherapeutic drug on endothelial cells, we set a threshold value of a $\eta$ and a dose per infusion of $q$ for reaching the cure state in the continuous model.

We consider chemotherapy as a forcing action on the cells in order to analyse their response to the treatment. Its time evolution, for a fixed infusion $q$, is given by:

$$
Q(t)=\frac{q}{\lambda}[1-\exp (-\lambda t)]
$$

Our aim is to investigate, for a fixed value of $q$, the effect of parameter $\eta$ related to the efficiency of the chemotherapeutic drug in killing the endothelial cells which affects the carrying capacity of tumour cells. Thus, we proceed with stability analysis for constant treatment. When $q(t)=q>0$, system (14) becomes autonomous and it has four equilibrium points:

- $P_{1}(0,0,0, q / \lambda)$-Elimination of normal and tumour cells due to high dose;

- $P_{2}\left(0, \widehat{N}_{2}, 0, q / \lambda\right)$-Curing disease through continuous chemotherapy;

- $P_{3}\left(\widehat{N}_{1}, 0, \widehat{L}_{1}, q / \lambda\right)$-Elimination of normal cells by treatment in the presence of tumour cells and vascular endothelial cells;

- $P_{4}\left(\widetilde{N}_{1}, \widetilde{N}_{2}, \widetilde{L}_{1}, q / \lambda\right)$-Coexistence between normal and tumour cells in the presence of vascular endothelial cells.

Let us consider the details for the cure state $P_{2}\left(0, \widehat{N}_{2}, 0, q / \lambda\right)$ :

$$
\widehat{N}_{2}=\frac{k_{2}\left(r_{2} b \lambda+q\left(r_{2}-v\right)\right)}{r_{2}(b \lambda+q)} .
$$

From a biological point of view, we have $\widehat{N}_{2}>0$, which occurs if

$$
0<q<q_{\text {threshold }} \text { with } v>r_{2},
$$

where

$$
q_{\text {threshold }}=\frac{r_{2} b \lambda}{v-r_{2}},
$$

corresponds to the rate of infusion of the drug required to cure.

The stability study of $P_{1}(0,0,0, q / \lambda)$ and $P_{2}\left(0, \widehat{N}_{2}, 0, q / \lambda\right)$ is given in the Appendix. In this analysis, we show that condition (27) guarantees the unstable character of $P_{1}(0,0,0, q / \lambda)$. The cure state $P_{2}\left(0, \widehat{N}_{2}, 0, q / \lambda\right)$ is locally stable when conditions (A.12) and (A.22), in the Appendix, hold:

(i) Condition (A.12), obtained in the Appendix and rewritten below, sets up a lower bound for the intensity of antiangiogenic effect of a chemotherapeutic drug, modelled by the parameter $\eta$ :

$$
\eta_{\text {threshold }}=\sigma\left(1+\frac{c \lambda}{q}\right) \text {, }
$$

below that, there is no cure for the disease. There is a transcritical bifurcation in the bifurcation diagram, assuming $\eta$ to be a control parameter, since $P_{2}$ changes from unstable to stable equilibrium. Therefore, above $\eta_{\text {threshold }}$, the tumour is eliminated. 


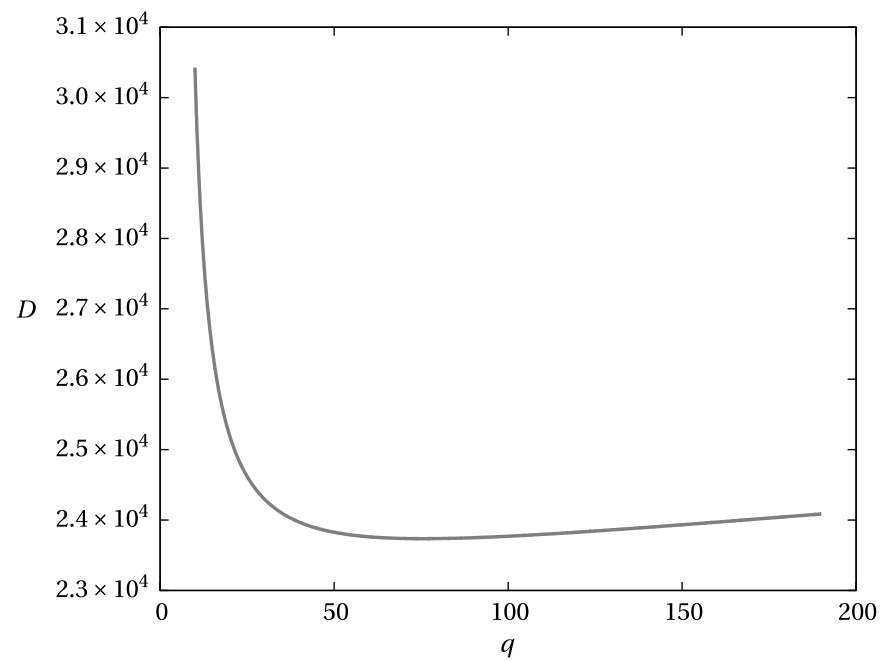

Fig. 6. Total chemotherapy dose $D$ as a function of the infusion rate $q$. The optimized minimum dose is obtained at $q \approx 75.7 \mathrm{mg} / \mathrm{day}$ and for which the time elapsed until cure is about 313 days. Parameter values are presented in Tables 1 and 2 , with $\eta=500$ day $^{-1}$. In order to get such a curve, tumoural elimination was conveniently defined here as a "tumour" with less than one cell. Initial condition: $N_{1}(0)=2 \times 10^{10}, N_{2}(0)=1 \times 10^{12}, L_{1}(0)=1 \times 10^{2}$ and $Q(0)=0$.

(ii) Condition (A.22), obtained in the Appendix and rewritten here as

$$
\alpha_{1}>\Lambda_{5} / \Lambda_{4}
$$

where

$$
\begin{aligned}
& \Lambda_{4}=-r_{1} k_{2}(a \lambda+q)\left(r_{2}(b \lambda+q)-v q\right), \\
& \Lambda_{5}=-r_{2} k_{1}(b \lambda+q)\left(r_{1}(a \lambda+q)-\mu q\right),
\end{aligned}
$$

sets up an inequality expressed in terms of the infusion rate $q$, which is, from a clinical perspective, a parameter that can be controlled.

In summary, the critical parameters $q$ and $\eta$, given, respectively by (28) and (29) correspond to the upper and lower values for the cure state; condition (A.21) is also necessary to guarantee the local stable character of the cure state.

Besides the steady state itself, we estimate the length of time to reach the cure state $t_{\text {cure }}$ by continuous drug administration, assuming the values of parameters in Tables 1 and $2, \eta=500$ day $^{-1}$ (as in the metronomic schedules). For this purpose, the constant infusion rate parameter $q$ was chosen by an optimization process because it can be controlled clinically. Namely, it is the value for which the total dose $D=q t_{\text {cure }}$ is minimum but still eliminates the tumour. It is important to note that $t_{\text {cure }}$ is a function of $q$ and then $D$ is nonlinear in $q$. For the chosen parameters, the total dose $D$ is shown as a function of $q$ in Fig. 6, from where one can see that $D$ reaches its minimum between 50 and 100 or, more precisely at $q \approx 75.7 \mathrm{mg} /$ day. Adopting this value for $q$, we finally estimate that the time to reach the tumoural elimination (cure state) is $\approx 313$ days. The larger the drug infusion rate $q$, the shorter the time $t_{\text {cure }}$ will be. If considering $q \approx 75.7 \mathrm{mg} /$ day the cure state can be reached with the smallest possible amount of chemotherapy.

\section{Concluding remarks}

In following with our main objective - to investigate the net antiangiogenic effect of metronomic chemotherapy in the vascular stage from the mathematical modelling point of view - in the current paper, through a simple mathematical model, we present how the antiangiogenic effect of metronomic chemotherapy takes place. We adopt a mean field approach, which lacks of spatial heterogeneity and the specifying details of endothelial cells interaction with the vessels and other cells in the neighbourhood of basal lamina (see Levine et al. [30]). Hopefully, however, we have quantified the antiangiogenic effect of metronomic chemotherapy by means of a computational approach.

According to our results, more frequent low doses of metronomic chemotherapy enhance tumoural reduction if the chemotherapeutic drug actually promotes an antiangiogenic effect on endothelial cells $(\eta \neq 0)$. Although there is a huge discussion about the efficiency of metronomic schedules in reducing the tumour, as far as we know, no one has been able to describe how this could happen. Here, we address this issue, first computing the antiangiogenic effect on endothelial cells and then exhibiting a counter-example for which decreasing the cycle time interval between drug infusions does not imply a smaller tumour when the antiangiogenic effect does not exist. Accordingly, we point out some questions: 'How can we take advantage of more frequent low-doses of metronomic chemotherapy?' If such a protocol is used, our investigation corroborates that the chemotherapeutic effect on endothelial cells entails an additional tumoural reduction. 
Analysing conventional and metronomic chemotherapy schedules with $(\eta \neq 0)$ and without $(\eta=0)$ an antiangiogenic effect on endothelial cells comparatively, and the continuous administration as a limit case of administration in cycles with the focus on the cure state, we can summarize our conclusions with the following items:

(a) Without any time delay in the equations, the model presented here describes the time lag between tumour cells and endothelial cells associated with "angiogenic switch" as reported by Folkman [17]. Moreover, our model indicates that the increasing of the tumour only occurs by the augmentation of vascularization.

(b) The killing action of chemotherapeutic drug on vascular endothelial cells (controlled by parameter $\eta$ in our model) is more relevant to the reduction of a tumour than the metronomic schedule of chemotherapy. However, applying a metronomic schedule for a chemical efficient in killing endothelial cells enhances the antiangiogenic effect, leading to a more effective tumour reduction.

(c) Increasing the total dose (for an almost non-cytotoxic chemotherapeutic drug effect on normal cells) is a better strategy for increasing the survival of a patient (after the total treatment time) for conventional or metronomic schedules. However, for metronomic schedules, at the end of treatment, larger doses are more effective for tumoural reduction.

(d) In the limit case of continuous drug administration, there are critical values for dose per infusion and chemotherapeutic action on endothelial cells that guarantee the elimination of cancer cells. Additionally, by a numerical example, we provide an estimate for the infusion rate which implies the smallest possible amount of chemotherapy, or total dose capable of eliminating the tumour. An infusion rate below such an optimal value implies more time to reach the cure, and then entails an increasing of the total dose. On the other hand, a higher infusion rate than the optimal eliminates the tumour more quickly, but higher doses. Using the optimal dose value, we present the time elapsed to reach the cure in the situation of continuous drug administration.

Finally, we point out that many other questions related to metronomic chemotherapy may be raised based on our approach, expanding our model, for instance, to consider the effect of frequent, low doses when the chemotherapeutic drug is combined with an antiangiogenic drug that acts only on endothelial cells $[12,16]$.

\section{Acknowledgements}

The authors gratefully acknowledge $\mathrm{H}$. Freedman for the discussions about cancer modelling and the anonymous referees for their valuable comments and constructive criticisms about the manuscript. DSR: PNPD/CAPES. PFAM: \#2013/08133-0, São Paulo Research Foundation (FAPESP); \#2009/15098-0 São Paulo Research Foundation(FAPESP); FUNDUNESP: 1886/00913. STRP: CNPq (contract no. 305176/2009-9), FAPESB (contract no. PNX 0006/2009) and INCT-CITECS (contract no. 57386/2008-9).

\section{Appendix. Stability analysis: continuous infusion model}

We present the stability analysis of the treatment model for continuous chemotherapy (system $(14)$ for $q(t)=q>0$ ). We have the Jacobian matrix $\mathbb{J}\left(N_{1}, N_{2}, L_{1}, Q\right)$ of system (14) given by

$$
\mathbb{J}\left(N_{1}, N_{2}, L_{1}, Q\right)=\left[\begin{array}{cccc}
j_{11} & j_{12} & j_{13} & j_{14} \\
j_{21} & j_{22} & 0 & j_{24} \\
j_{31} & 0 & j_{33} & j_{34} \\
0 & 0 & 0 & -\lambda
\end{array}\right],
$$

where

$$
\begin{aligned}
& j_{11}=\frac{r_{1}\left(k_{1}-2 N_{1}-\alpha_{1} N_{2}\right)}{k_{1}+L_{1}}-\frac{\mu Q}{a+Q}, \quad j_{22}=\frac{r_{2}\left(k_{2}-2 N_{2}-\alpha_{2} N_{1}\right)}{k_{2}}-\frac{v Q}{b+Q}, \\
& j_{12}=-\frac{r_{1} N_{1} \alpha_{1}}{k_{1}+L_{1}}, \quad j_{21}=-\frac{r_{2} N_{2} \alpha_{2}}{k_{2}}, \quad j_{13}=\frac{r_{1} N_{1}\left(N_{1}+\alpha_{1} N_{2}\right)}{\left(k_{1}+L_{1}\right)^{2}}, \\
& j_{14}=-\frac{\mu N_{1}}{a+Q}+\frac{\mu N_{1} Q}{(a+Q)^{2}}, \quad j_{24}=-\frac{v N_{2}}{b+Q}+\frac{v N_{2} Q}{(b+Q)^{2}}, \\
& j_{34}=-\frac{\eta L_{1}}{c+Q}+\frac{\eta L_{1} Q}{(c+Q)^{2}}, \quad j_{31}=\phi-\omega L_{1}, \quad j_{33}=\sigma-\omega N_{1}-\frac{\eta Q}{c+Q} .
\end{aligned}
$$

Evaluated in $P_{1}(0,0,0, q / \lambda)$, the Jacobian matrix is given by

$$
\mathbb{J}(0,0,0, q / \lambda)=\left[\begin{array}{cccc}
\frac{r_{1} a \lambda+q\left(r_{1}-\mu\right)}{a \lambda+q} & 0 & 0 & 0 \\
0 & \frac{r_{2} b \lambda+q\left(r_{2}-v\right)}{b \lambda+q} & 0 & 0 \\
0 & 0 & \frac{\sigma c \lambda+q(\sigma-\eta)}{c \lambda+q} & 0 \\
0 & 0 & 0 & -\lambda
\end{array}\right],
$$


and the eigenvalues are

$$
\begin{aligned}
& \Omega_{1}=\frac{r_{1} a \lambda+q\left(r_{1}-\mu\right)}{a \lambda+q}, \\
& \Omega_{2}=\frac{r_{2} b \lambda+q\left(r_{2}-v\right)}{b \lambda+q}, \\
& \Omega_{3}=\frac{\sigma c \lambda+q(\sigma-\eta)}{c \lambda+q}, \\
& \Omega_{4}=-\lambda .
\end{aligned}
$$

Biologically, the equilibrium point $P_{1}(0,0,0, q / \lambda)$ has to be unstable. As $\lambda>0$, then $\Omega_{4}<0$. Condition (27) is such that $\Omega_{2}>0$, so it guarantees the unstable character of $P_{1}(0,0,0, q / \lambda)$.

Let us now see under what conditions the equilibrium point $P_{2}\left(0, \widehat{N}_{2}, 0, q / \lambda\right)$ (cure via treatment) is stable. Evaluated in $P_{2}\left(0, \widehat{N}_{2}, 0, q / \lambda\right)$, the Jacobian matrix becomes

$$
\mathbb{J}\left(0, \widehat{N}_{2}, 0, q / \lambda\right)=\left[\begin{array}{cccc}
p_{11} & 0 & 0 & 0 \\
-\frac{r_{2} \widehat{N}_{2} \alpha_{2}}{k_{2}} & p_{22} & 0 & -\frac{v \widehat{N}_{2} \lambda^{2} b}{(b \lambda+q)^{2}} \\
\phi & 0 & \frac{\sigma c \lambda+q(\sigma-\eta)}{c \lambda+q} & 0 \\
0 & 0 & 0 & -\lambda
\end{array}\right],
$$

where

$$
p_{11}=\left[r_{1}(a \lambda+q)\left(k_{1}-\alpha_{1} \widehat{N}_{2}\right)-\mu q k_{1}\right] /\left[k_{1}(a \lambda+q)\right]
$$

and

$$
p_{22}=\left[r_{2}(b \lambda+q)\left(k_{2}-2 \widehat{N}_{2}\right)-v q k_{2}\right] /\left[k_{2}(b \lambda+q)\right] .
$$

Substituting $\widehat{N}_{2}=\left[k_{2}\left(r_{2} b \lambda+q\left(r_{2}-v\right)\right)\right] /\left[r_{2}(b \lambda+q)\right]$ in $p_{11}$ and $p_{22}$, we obtain the eigenvalues of $\mathbb{J}\left(0, \widehat{N}_{2}, 0, q / \lambda\right)$, which are denoted by $\theta_{i}(i=1,2,3,4)$, given by

$$
\begin{aligned}
& \theta_{1}=\frac{r_{1} a \lambda+q\left(r_{1}-\mu\right)}{a \lambda+q}-\frac{\alpha_{1} r_{1}\left[k_{2}\left(r_{2} b \lambda+q\left(r_{2}-v\right)\right)\right]}{k_{1} r_{2}(b \lambda+q)}, \\
& \theta_{2}=-\frac{\left[r_{2} b \lambda+q\left(r_{2}-v\right)\right]}{b \lambda+q}, \\
& \theta_{3}=\frac{\sigma c \lambda+q(\sigma-\eta)}{c \lambda+q}, \\
& \theta_{4}=-\lambda .
\end{aligned}
$$

Therefore $\theta_{2}=-\Omega_{2}, \theta_{3}=\Omega_{3}$ and $\theta_{4}=\Omega_{4}$. For the stability conditions of $P_{2}\left(0, \widehat{N}_{2}, 0, q / \lambda\right)$, we have $\theta_{4}<0$; if the inequality (27) is satisfied, $\theta_{2}<0$. The eigenvalue $\theta_{3}$ is negative if

$$
\eta>\frac{\sigma(c \lambda+q)}{q},
$$

i.e., there is a lower bound for the intensity of the antiangiogenic effect below which there is no cure for the disease. This value is given by (A.12).

The eigenvalue $\theta_{1}$ may be re-written as

$$
\theta_{1}=\frac{\Lambda_{1} q^{2}+\Lambda_{2} q+\Lambda_{3}}{k_{1} r_{2}(a \lambda+q)(b \lambda+q)},
$$

where

$$
\begin{aligned}
& \Lambda_{1}=r_{2} k_{1}\left(r_{1}-\mu\right)+\alpha_{1} k_{2} r_{1}\left(v-r_{2}\right), \\
& \Lambda_{2}=(a+b)\left[r_{1} r_{2} \lambda\left(k_{1}-\alpha_{1} k_{2}\right)\right]+\lambda\left(r_{1} a v \alpha_{1} k_{2}-r_{2} b \mu k_{1}\right), \\
& \Lambda_{3}=r_{1} r_{2} a b \lambda^{2}\left(k_{1}-\alpha_{1} k_{2}\right) .
\end{aligned}
$$


For the equilibrium $P_{2}\left(0, \widehat{N}_{2}, 0, q / \lambda\right)$ to be locally asymptotically stable, condition (27) must be satisfied and $\theta_{3}$ has to be negative. Thus, using (A.13), we have

$$
\frac{\Lambda_{1} q^{2}+\Lambda_{2} q+\Lambda_{3}}{k_{1} r_{2}(a \lambda+q)(b \lambda+q)}<0 \quad \Longrightarrow \quad \Lambda_{1} q^{2}+\Lambda_{2} q+\Lambda_{3}<0 .
$$

Substituting (A.14)-(A.16) in (A.17), we have

$$
\Lambda_{4} \alpha_{1}-\Lambda_{5}<0
$$

where

$$
\begin{aligned}
& \Lambda_{4}=-r_{1} k_{2}(a \lambda+q)\left(r_{2}(b \lambda+q)-v q\right), \\
& \Lambda_{5}=-r_{2} k_{1}(b \lambda+q)\left(r_{1}(a \lambda+q)-\mu q\right),
\end{aligned}
$$

resulting

$$
\Lambda_{4} \alpha_{1}<\Lambda_{5}
$$

From (27), we have $\Lambda_{4}<0$ and we get

$$
\alpha_{1}>\frac{\Lambda_{5}}{\Lambda_{4}}
$$

If $\Lambda_{5}>0$, then $\Lambda_{5} / \Lambda_{4}<0$ and inequality (A.22) always holds, since $\alpha_{1} \geq 0$. On the other hand, if $\Lambda_{5}<0$, then $\Lambda_{5} / \Lambda_{4}>0$ and inequality (A.22) must be satisfied for $P_{2}\left(0, N_{2}, 0, q / \lambda\right)$ to be stable.

\section{References}

[1] J.C. Panetta, K.R. Fister, Optimal control applied to competing chemotherapeutic cell-kill strategies, SIAM J. Appl. Math. 63 (6) (2003) 1954-1971.

[2] R.A. Gatenby, Application of competition theory to tumour growth: implications for tumour biology and treatment, Eur. J. Cancer 32A (1996) $722-726$.

[3] H.M. Byrne, M.A.J. Chaplain, Growth of nonnecrotic tumours in the presence and absence of inhibitors, Math. Biosci. 130 (2) (1995) $151-181$.

[4] M.A.J. Chaplain, S.R. McDougall, A.R.A. Anderson, Mathematical modelling of tumour-induced angiogenesis, Annu. Rev. Biomed. Eng. 8 (2006) $233-257$.

[5] J. Moreira, A. Deutsch, Cellular automaton models of tumour development: a critical review, Adv. Complex Syst. 5 (2-3) (2002) $247-267$.

[6] G.S. Stamatakos, E.A. Kolokotroni, D.D. Dionysiou, E.C. Georgiadi, C. Desmedt, An advanced discrete state-discrete event multiscale simulation model of the response of a solid tumour to chemotherapy: mimicking a clinical study, J. Theoret. Biol. 266 (1) (2010) 124-139.

[7] P. Macklin, S. McDougall, A.R.A. Anderson, M.A.J. Chaplain, V. Cristini, J. Lowengrub, Multiscale modelling and nonlinear simulation of vascular tumour growth, J. Math. Biol. 58 (4-5) (2009) 765-798.

[8] M.R. Owen, T. Alarcon, P.K. Maini, H.M. Byrne, Angiogenesis and vascular remodelling in normal and cancerous tissues, J. Math. Biol. 58 (2009) 689-721.

[9] N.L. Komarova, Mathematical modelling of tumourigenesis: mission possible, Curr. Opin. Oncol. 17 (1) (2005) 39-43.

[10] H.M. Byrne, T. Alarcon, M.R. Owen, S.D. Webb, P.K. Maini, Modelling aspects of cancer dynamics: a review, Phil. Trans. R. Soc. A 364 (2006) $1563-1578$.

[11] R.P. Araujo, D.L.S. McElwain, A history of the study of solid tumour growth: the contribution of mathematical modelling, Bull. Math. Biol. 66 (2004) $1039-1091$.

[12] T. Browder, C.E. Butterfield, B.M. Kraling, B. Shi, B. Marshall, M.S. O'Reilly, J. Folkman, Antiangiogenic scheduling of chemotherapy improves efficacy against experimental drug-resistant cancer, Cancer Res. 60 (2000) 1878-1886.

[13] D. Hanahan, G. Bergers, E. Bergsland, Less is more, regularly: metronomic dosing of cytotoxic drugs can target tumour angiogenesis in mice, J. Clin. Invest. 105 (2000) 1045-1047.

[14] R.S. Kerbel, B.A. Kamem, The anti-angiogenic basis of metronomic chemotherapy, Nat. Rev. Cancer 4 (2004) 423-436.

[15] P. Hahnfeldt, D. Panigrahy, J. Folkman, L. Hlatky, Tumour development under angiogenic signaling: a dynamical theory of tumour growth treatment response, and postvascular dormancy, Cancer Res. 59 (1999) 4770-4775.

[16] S.T.R. Pinho, F.S. Bacelar, R.F.S. Andrade, H.I. Freedman, A mathematical model for the effect of anti-angiogenic therapy in the treatment of cancer tumours by chemotherapy, Nonlinear Anal.-Real 14 (2013) 815-828.

[17] J. Folkman, Tumour angiogenesis: therapeutic implications, N. Engl. J. Med. 285 (21) (1971) 1182-1186.

[18] A. Bikfalvi, Significance of angiogenesis in tumour progression and metastasis, Eur. J. Cancer 31A (1995) 1101-1104.

[19] J. Folkman, Role of angiogenesis in tumour growth and metastasis, Semin. Oncol. 29 (6(suppl. 16)) (2002) 15-18.

[20] F. Bussolino, M. Arese, E. Audeo, E. Giraudo, S. Marchió, S. Mitola, L. Primo, G. Serini, Biological aspects of tumour angiogenesis, in: L. Preziosi (Ed.), Cancer Modelling and Simulation, Chapman \& Hall/CRC, London, 2003, pp. 1-22.

[21] M.S. O’Reilly, T. Boehm, Y. Shing, N. Fukai, G. Vasios, W.S. Lane, E. Flynn, J.R. Birkhead, B.R. Olsen, J. Folkman, Endostastin: an endogenous inhibitor of angiogenesis and tumour growth, Cell 88 (1997) 1-20.

[22] L. Bello, G. Carrabba, C. Giussani, V. Lucini, F. Cerutti, F. Scaglione, J. Landé, M. Pluderi, G. Tomei, R. Villani, R.S. Carroll, P. McLBlack, A. Bikfalvi, Low-dose chemotherapy combined with an antiangiogenic drug reduces human glioma growth in vivo, Cancer Res. 61 (20)(2001) 7501-7506.

[23] S. Man, G. Bocci, G. Francia, S.K. Green, S. Jothy, D. Hanahan, P. Bohlen, D.J. Hicklin, G. Bergers, R.S. Kerbel, Antitumour effects in mice of low-dose (metronomic) cyclophosphamide administered continuously through the drinking water, Cancer Res. 62 (10)(2002) 2731-2735.

[24] S. Baruchel, D. Stempak, Low-dose metronomic chemotherapy: myth or truth? Onkologie 29 (1996) 305-307.

[25] K. Lien, S. Georgsdottir, L. Sivanathan, K. Chan, U. Emmenegger, Low-dose metronomic chemotherapy: a systematic literature analysis, Eur. J. Cancer 49 (16) (2013) 3387-3395.

[26] A. d'Onofrio, A. Gandolfi, Tumour eradication by antiangiogenic therapy: analysis and extensions of the model by Hahnfeldt et. al. (1999), Math. Biosci. 191 (2004) 159-184.

[27] A. d'Onofrio, A. Gandolfi, A family of models of angiogenesis and anti-angiogenesis anti-cancer therapy, Math. Med. Biol. 26 (2009) 63-95.

[28] D.S. Rodrigues, S.T.R. Pinho, P.F.A. Mancera, Um modelo matemático em quimioterapia, TEMA 13 (1) (2012) 1-12 (in portuguese).

[29] S.A. Maggelakis, The effects of stimulator and inhibitor factors on tumour vascularization: a mathematical model, Math. Comput. Modelling 23 (6) (1996) $121-133$.

[30] H.A. Levine, B.D. Sleeman, M. Nilsen-Hamilton, Mathematical model for the roles of pericytes and macrophages in the initiation of angiogenesis I: the role of protease inhibitors in preventing angiogenesis, Math. Biosci. 168 (2000) 77-115.

[31] P. Hahnfeldt, J. Folkman, L. Hlatky, Minimizing long-term tumour burden: the logic for metronomic chemotherapeutic dosing and its antiangiogenic basis, J. Theoret. Biol. 220 (4) (2003) 545-554. 
[32] C. Phipps, Combination of chemotherapy and antiangiogenic therapies: a mathematical modelling approach (M.Sc. dissertation), University of Waterloo, Waterloo, 2009.

[33] S.T.R. Pinho, H.I. Freedman, F.K. Nani, A chemotherapy model for the treatment of cancer with metastasis, Math. Comput. Modelling 36 (2002) $773-803$.

[34] F. Nani, H.I. Freedman, A mathematical model of cancer treatment by immunotherapy, Math. Biosci. 163 (4)(2000) 159-199.

[35] S.T.R. Pinho, D.S. Rodrigues, P.F.A. Mancera, A mathematical model of chemotherapy response to tumour growth, Can. Appl. Math. Q. 19 (4) (2011) $369-384$.

[36] R. Martin, K.L. Teo, Optimal Control of Drug Administration in Cancer Chemotherapy, World Scientific, Singapore, 1993.

[37] M. Retsky, Letter to the editor, J. Theoret. Biol. 229 (3) (2004) 289.

[38] J.S. Spratt, J.S. Meyer, J.A. Spratt, Rates of growth of human neoplasms: part II, J. Surg. Oncol. 61 (2) (1996) 68-73.

[39] Z. Bajzer, S.V. Pavlovic, Mathematical modelling of tumour growth kinetics, in: J.A. Adam, N. Bellomo (Eds.), A Survey of Models for Tumour-Immune System Dynamics, Birkhäuser, Boston, 1997, pp. 126-127.

[40] S. Michelson, A.S. Glicksman, J.T. Leith, Growth in solid heterogeneous human colon adenocarcinomas: comparison of simple logistical models, Cell Prolif. 20 (3) (1987) 343-355.

[41] V.G. Vaidya, F.J. Alexandro Jr., Evaluation of some mathematical models for tumour growth, Int. J. Bio-Med. Comput. 13 (1) (1982) 19-35.

[42] D. Hanahan, R.A. Weinberg, Hallmarks of Cancer: the next generation, Cell 144 (5) (2011) 646-674.

[43] M. Boareto, M.K. Jolly, E. Ben-Jacob, J.N. Onuchic, Jagged mediates differences in normal and tumor angiogenesis by affecting tip-stalk decision, Proc Natl. Acad. Sci. 112 (29) (2015) E3836-E3844.

[44] H. Lüllmann, K. Mohr, A. Ziegler, D. Bieger, Color Atlas of Pharmacology, Thieme, Stuttgart, 2000.

[45] H.E. Skipper, F.M. Schaebel Jr., W.S. Wilcox, Experimental evaluation of potential anticancer agents XIII: on the criteria and kinetics associated with curability of experimental leukemia, Cancer Chemother. Rep. 35 (1964) 1-111.

[46] J. Aroesty, T. Lincoln, N. Shapiro, G. Boccia, Tumour growth and chemotherapy: mathematical methods, computer simulations, and experimental foundations, Math. Biosci. 17 (1973) 243-300.

[47] D.S. Rodrigues, Modelagem matemática em câncer: dinâmica angiogênica e quimioterapia anti-neoplásica (M.Sc. dissertation), 2011 (in portuguese)

[48] R.N. Buick, Cellular basis of chemotherapy, in: R.T. Dorr, D.D. Von Hoff (Eds.), Cancer Chemotherapy Handbook, Appleton \& Lange, Norwalk, 1994 pp. 3-14.

[49] R.S. Kerbel, Tumour angiogenesis: past, present and the near future, Carcinogenesis 21 (3) (2000) 505-515.

[50] R.A. Weinberg, The Biology of Cancer, Garland Science, New York, 2006.

[51] F.M. Schaebel Jr., Concepts for systematic treatment of micrometastases, Cancer 35 (1975) 15-24.

[52] U. Monte, Does the cell number $10^{9}$ still really fit one gram of tumor tissue? Cell Cycle 8 (3) (2009) 505-506.

[53] E. Bianconi, A. Piovesan, F. Facchin, A. Beraudi, Raffaella Casadei, F. Frabetti, L. Vitale, M.C. Pelleri, S. Tassani, F. Piva, S. Perez-Amodio, P. Strippoli, S. Canaider, An estimation of the number of cells in the human body, Ann. Hum. Biol. 40 (6) (2013) 463-471.

[54] MeadJohnson Oncology Products [internet] accessed 03.08.11. Available from http://patient.cancerconsultants.com/druginserts/Cyclophosphamide. pdf.

[55] FEC100 chemotherapy for breast cancer (Written by Jeremy Braybrooke). Document number: ASWCS09 BR006 [internet] accessed 27.07.11. Available from http://www.avon.nhs.uk/aswcs-chemo/STCP/index.htm.

[56] R.D. Mosteller, Simplified calculation of body surface area, N. Engl. J. Med. (1987) 1098.

[57] S.E. Singletary, C. Allred, P. Ashley, L.W. Bassett, D. Berry, K.I. Bland, P.I. Borgen, G. Clark, S.B. Edge, D.F. Hayes, L.L. Hughes, R.V. Hutter, M. Morrow, D.L. Page, A. Recht, R.L. Theriault, A. Thor, D.L. Weaver, H.S. Wieand, F.L. Greene, Revision of the American joint committee on cancer staging system for breast cancer, J. Clin. Oncol. 20 (17) (2002) 3628-3636. 